\title{
Relation between coronary plaque calcium deposits as described by computed tomography coronary angiography and acute results of stent deployment as assessed by intravascular ultrasound
}

\author{
Jerzy Pregowski, Jan Jastrzebski, Cezary Kępka, Mariusz Kruk, Michał Ciszewski, Rafał Wolny, Joanna Zalewska, \\ Zbigniew Chmielak, Maciej Karcz, Adam Witkowski
}

Institute of Cardiology, Warsaw, Poland

Postep Kardiol Inter 2013; 9, 2 (32): 115-120

DOI: $10.5114 /$ pwki.2013.35444

\begin{abstract}
Introduction: The findings from intravascular ultrasound studies on the impact of calcium deposits on the results of stent implantation are conflicting.

Aim: To evaluate whether calcium deposits as assessed by (CTCA) influence results of stent deployment.

Material and methods: The study population comprised 60 patients ( 43 male; age $64.2 \pm 8.6$ years) who underwent CTCA before stent implantation. Lesion calcium score, total calcium length, and maximal area and maximal thickness of calcium deposits within the lesion segment were assessed. Plaques were divided into those with calcium score $\geq$ median (group 1), calcium score $<$ median (group 2), and without calcium (group 3). Intravascular ultrasound (IVUS) was performed after attainment of optimal angiographic results of the stent procedure. Focal and diffuse stent expansion was defined as either minimum stent area (MSA) or mean stent area over the length of the stent divided by reference lumen area.

Results: The proximal reference segments of lesions with higher calcium score contained a larger plaque burden ( $47 \pm 12 \%$ vs. 41 $\pm 9 \%$ vs. $34 \pm 18 \%, p=0.02$ ) - respectively for groups 1 , 2 , and 3 . Positive correlation was observed between lesion calcium score and frequency of post-dilation ( $R=0.28, p=0.03)$. There was no difference in focal stent expansion $(71 \pm 14 \%$ vs. $65 \pm 15 \%$ vs. $71 \pm 15 \%$, $p=0.3$ ) or diffuse stent expansion ( $92 \pm 30 \%$ vs. $85 \pm 30 \%$ vs. $93 \pm 38 \%, p=0.7$ ) comparing groups 1 , 2, and 3 . Lesion calcium score, total length of calcium, and maximum area and thickness of calcium deposits did not correlate with focal or diffuse stent expansion.

Conclusions: Lesions with a higher CTCA calcium score had larger reference plaque burden after stent implantation and more likely required post-dilation, but final stent expansion as assessed by IVUS was not affected by the amount of CTCA calcium provided an angiographically optimal result was achieved.
\end{abstract}

Key words: computed tomography coronary angiography, calcium deposits.

\section{Introduction}

The findings from previous intravascular ultrasound studies on the relation between calcium deposits and results of stent deployment are conflicting [1-3]. Computed tomography coronary angiography (CTCA) allows detailed assessment of coronary plaque calcium including the features that cannot be quantified by intravascular ultrasound (IVUS): area and thickness of calcium deposits.

Aim
The aim of our study was to evaluate whether pres-
ence and quantitative characteristics of calcium deposits within coronary lesion as assessed by CTCA may influence the results of stent deployment evaluated with IVUS.

\section{Material and methods}

The current study was a post-hoc analysis of the results of a single center randomized study with IVUS endpoints [4]. The protocol of the initial study has been registered at ClinicalTrials.gov (NCT01205425). The detailed inclusion and exclusion criteria have been published [4]. All patients included in the current analysis underwent coronary stent implantation and IVUS examination after the operator confirmed an optimal angiographic outcome

Corresponding author:

Jerzy Pregowski MD, PhD, Institute of Cardiology, 42 Alpejska St, 04-628 Warsaw, Poland, tel.: +48 501472 421, e-mail: jerzypregowski74@gmail.com Received: 4.01.2013, accepted: 9.05.2013. 
of the procedure defined as a residual stenosis $<30 \%$ by visual estimation. The study was approved by the local Ethics Committee and was performed in accordance with the Helsinki II Declaration. All participating patients signed informed consent. We included consecutive patients with stable coronary artery diseases who underwent CTCA as the first anatomical evaluation of coronary arteries prior to invasive coronary angiography. The major inclusion criterion was the presence of at least one single significant ( $>70 \%$ stenosis) CTCA coronary lesion in a native coronary artery likely to undergo percutaneous coronary intervention $(\mathrm{PCl})$.

Prior to contrast enhanced CTCA a native scan was performed for the assessment of the calcium score (expressed as Agatston Score Equivalents [ASE]). Quantification of coronary calcifications was performed using dedicated software for post-processing (Syngo Multimodality Workplace $^{\mathrm{TM}}$, Siemens, Forchheim, Germany). An experienced observer (JJ) blinded to the IVUS data analyzed the calcium score scan in relation to the coronary angiogram recorded during percutanous coronary intervention ( $\mathrm{PCI})$. Then the value of the calcium score confined to the stented segment (lesion calcium score) was assessed to quantify the lesion-specific calcium deposits (Figure 1). Lesions were divided into three groups: no calcium, lesion calcium score $<$ median, and lesion calcium score $\geq$ median.

The contrast enhanced CTCA dataset was acquired with a dual-source CT scanner (Somatom Definition, Siemens, Forchheim, Germany) after sublingual administration of nitrates $(0.8 \mathrm{mg})$. The CTCA data were analyzed off-line with quantitative CT software (Circulation ${ }^{\circledR}$, Siemens, Forchheim, Germany). Images were displayed with a visu- ally adjusted setting and were analyzed by an experienced observer (J) simultaneously with the coronary angiogram. To differentiate calcium deposits from the contrast medium the scout scan was compared with the contrast enhanced CTCA. Several parameters featuring calcifications within the stented segment were measured: total length of all calcium deposits, maximal calcium area in a single cross-section, and maximal calcium thickness at the site of maximal calcium area (Figure 2).

The IVUS was performed after attaining optimal angiographic results. Intracoronary nitroglycerine (0.2 $\mathrm{mg}$ ) administration was performed, and the IVUS catheter (Volcano Corporation, San Diego, California, USA) was advanced $>10 \mathrm{~mm}$ distal to the stented segment; and imaging was performed retrograde with an automatic pullback $(0.5 \mathrm{~mm} / \mathrm{s})$. Off-line IVUS analyses were performed by an observer (JP) blinded to the CTCA findings and according to the established criteria [5]. Planimetric IVUS measurements were performed at the minimal stent area (MSA) site and at the proximal and distal reference sites defined as the largest lumen area located within $10 \mathrm{~mm}$ long segments adjacent to the stent shoulders, but before a major side-branch. Volumetric assessment of the stented segment was performed every $1 \mathrm{~mm}$ to calculate the mean stent area. Reference measurements included lumen and external elastic membrane (EEM) areas; plaque burden was calculated according to the formula: ([EEM area - lumen area]/EEM × 100\%). Focal stent expansion was defined as the MSA divided by mean reference lumen area. Diffuse stent expansion was defined as mean stent area divided by the mean reference lumen area. Quantitative IVUS findings including
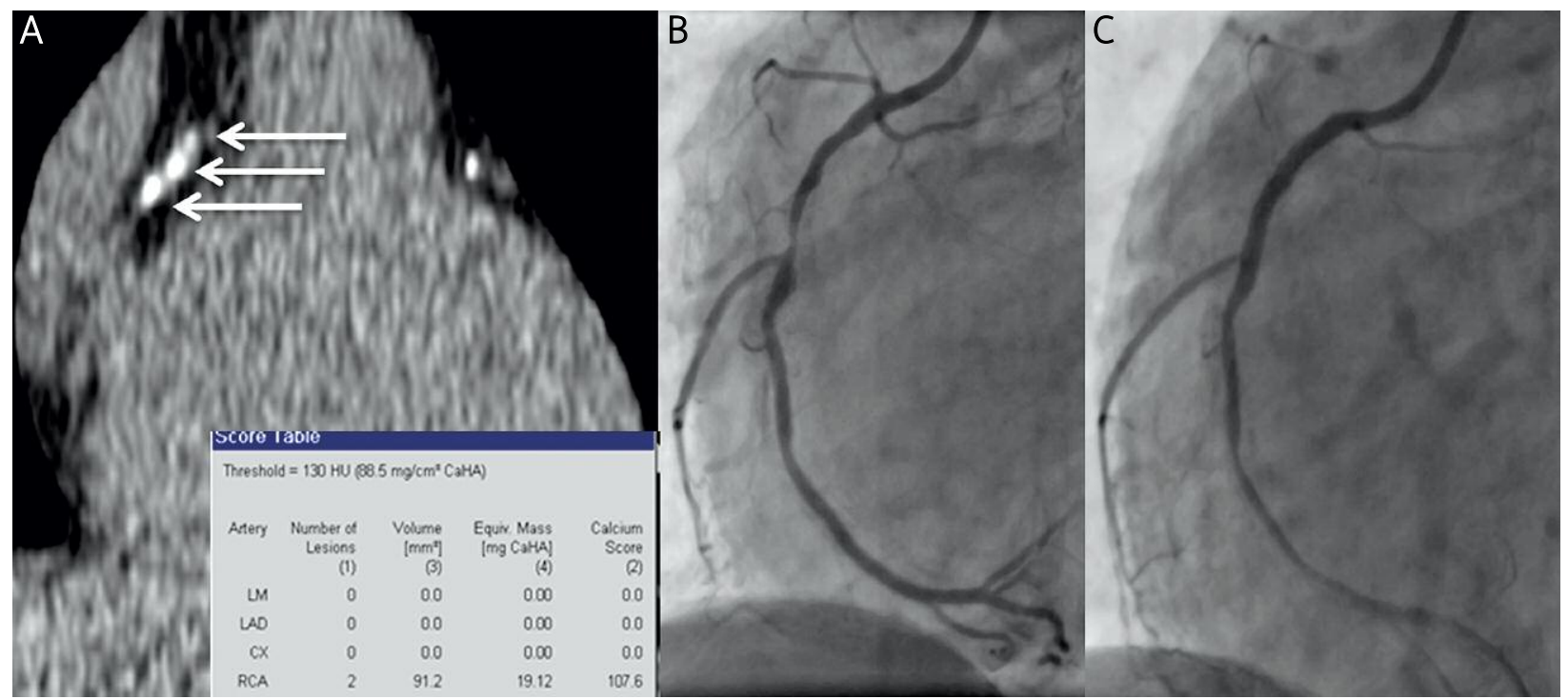

Fig. 1. The lesion calcium score scan in relation to the coronary angiogram recorded during PCl. A-Scout CT scan performed for the evaluation of calcium score. Arrows indicate calcium deposits located within the target lesion in the right coronary artery (lesion calcium score: 107.6). B - Coronary angiography with significant lesion at the location of calcium deposits in mid right coronary artery. C - Right coronary artery after stent implantation 


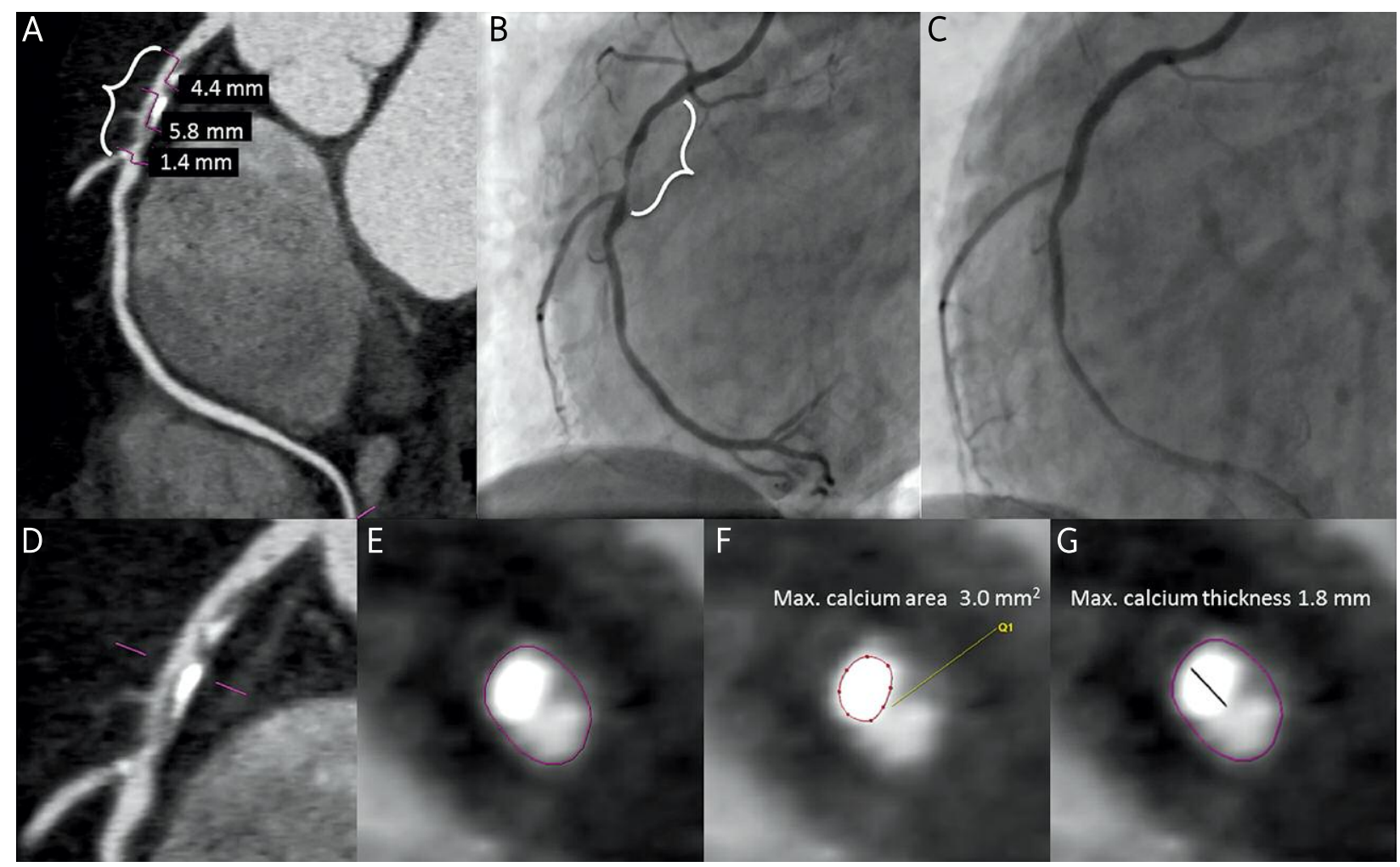

Fig. 2. Calcium measurements within the coronary lesion detected by CTCA. A - Curved multiplanar reconstruction of RCA with significant stenosis; three separate calcium deposits were identified within the lesion (total length: $11.6 \mathrm{~mm}$ ). B - The cineangiogram of RCA with stenosis initially detected by CTCA. C - RCA after stent implantation. D - Curved multiplanar reconstruction of RCA focused on the lesion site. E - The cross-section located within the target lesion with maximal area and thickness of calcium. F-Measurement of maximal area of calcium $(3.0 \mathrm{~mm})$. G - Measurement of maximal calcium thickness $(1.8 \mathrm{~mm})$

presence of stent malapposition, tissue prolapse, and stent edge dissection were identified according to established criteria [6-8].

\section{Statistical analysis}

Continuous data with normal distribution are presented as means with standard deviation while the non-normally distributed variables are presented as medians with interquartile ranges (IQR). An independent samples t-test, one-way ANOVA or Mann-Whitney test was used to assess differences between continuous variables. Categorical variables were compared with $\chi^{2}$ test. Correlations of continuous variables were assessed with the Spearman test. We used MedCalc version 9.3.8.0 (Mariakerke, Belgium) for statistical analyses.

\section{Results}

The study population comprised 60 patients in whom 64 separate coronary lesions were treated with stent implantation between September 2009 and April 2011. Paired native scan for calcium score assessment and contrast enhanced CTCA were available for 60 lesions (94\%); this group is the basis for all analyses performed. The medi- an time between CTCA and stent implantation was 30 (14.5-47.5) days. Calcium deposits were identified in 47 (78\%) lesions. In lesions containing calcium deposits, the median calcium score was 60.2 (10.9-152.2), median length of the calcium deposits was 7.5 (3.9-10.9) mm, median maximal calcium area was 3.1 (1.8-5.0) $\mathrm{mm}^{2}$, and median maximal calcium thickness was 1.5 (1.3-1.8) mm.

Baseline demographic, clinical and procedural variables of the study patients divided into groups according to the lesion calcium score are presented in Table 1. There was no significant difference between maximal balloon pressure during stent implantation into lesions without calcium, lesions with calcium score $<$ median, and lesions with calcium score $\geq$ median $(15.2 \pm 2.9 \mathrm{~atm}$ vs. $16.1 \pm 2.6 \mathrm{~atm}$ vs. $16.8 \pm 2.9 \mathrm{~atm}, p=0.2$ ). However, we observed a correlation between maximal calcium cross-sectional area within the stented segment and the maximal balloon pressure applied to achieve optimal angiographic results ( $R=0.32, p=0.01)$. Moreover, in order to obtain optimal angiographic results, stent post-dilation tended to be more often required in lesions with a higher calcium score: 9 (38\%) vs. 4 (17\%) vs. 1 (8\%), $p=0.09$, respectively, for lesions with calcium score $\geq$ median, lesions with calcium 
Table 1. Baseline demographic, clinical and procedural variables with regards to the lesion calcium score

\begin{tabular}{|c|c|c|c|c|}
\hline Variable & $\begin{array}{l}\text { Lesion calcium } \\
\text { score }=0(n=13)\end{array}$ & $\begin{array}{l}\text { Lesion calcium } \\
\text { score }<\text { median }(n=23)\end{array}$ & $\begin{array}{c}\text { Lesion calcium } \\
\text { score } \geq \text { median }(n=24)\end{array}$ & Value of $p$ \\
\hline Male, $n$ & 9 & 16 & 14 & 0.6 \\
\hline Age [years], mean \pm SD & $62.8 \pm 6.9$ & $64.2 \pm 8.5$ & $65.9 \pm 9.0$ & 0.6 \\
\hline Diabetes mellitus, $n$ & 3 & 5 & 3 & 0.7 \\
\hline Hypercholesterolemia, $n$ & 11 & 20 & 21 & 0.5 \\
\hline Hypertension, $n$ & 11 & 21 & 21 & 0.5 \\
\hline \multicolumn{4}{|l|}{ Location of target lesion, $n$} & \multirow[t]{4}{*}{0.5} \\
\hline LAD & 4 & 16 & 14 & \\
\hline LCX & 3 & 2 & 2 & \\
\hline RCA & 6 & 5 & 8 & \\
\hline Nominal stent diameter [mm] & $3.2 \pm 0.4$ & $3.2 \pm 0.5$ & $3.1 \pm 0.4$ & 0.9 \\
\hline Maximal balloon pressure [atm] & $15.2 \pm 2.9$ & $16.1 \pm 2.6$ & $16.8 \pm 2.9$ & 0.2 \\
\hline Length of the stented segment [mm] & $21.0 \pm 8.4$ & $19.8 \pm 4.8$ & $23.5 \pm 7.6$ & 0.15 \\
\hline Number of stents/lesion & $1.1 \pm 0.3$ & $1.1 \pm 0.3$ & $1.0 \pm 0.2$ & 0.5 \\
\hline Direct stenting, $n$ & 9 & 18 & 13 & 0.2 \\
\hline Post-dilation, $n$ & 1 & 4 & 9 & 0.09 \\
\hline
\end{tabular}

score $<$ median, and lesions without calcium. A positive correlation was observed between increasing lesion calcium score and frequency of post-dilation $(R=0.28, p=0.03)$.

Calcium containing lesions were located in larger vessels as indicated by the difference in the proximal reference EEM area $\left(18.2 \pm 4.5 \mathrm{~mm}^{2}\right.$ vs. $19.8 \pm 5.9 \mathrm{~mm}^{2}$ vs. 14.4 $\pm 4.3 \mathrm{~mm}^{2}, p=0.03$, respectively, for lesions with calcium score $\geq$ median, lesions with calcium score $<$ median, and lesions without calcium). With increasing lesion site calcium, we observed a larger plaque burden in the reference segments adjacent to the proximal stent edge $(p=0.02)$ (Table 2).

The MSA and mean stent area were similar regardless of the magnitude of lesion calcium (Table 2). The percentage of lesions with MSA $<4.5 \mathrm{~mm}^{2}$ did not differ between the groups: $6(25 \%)$ vs. $6(26 \%)$ vs. $3(23 \%), p=0.9$, respectively for lesions with calcium score $\geq$ median, lesions with calcium score $<$ median, and lesions without calcium. Total length of calcium deposits, maximal calcium area, and thickness did not affect MSA (Table 3). There was no significant difference in focal stent expansion between lesions categorized according to their calcium score: $71 \pm 14 \%$ vs. $65 \pm 15 \%$ vs. $71 \pm 15 \%, p=0.3$, respectively, for lesions with calcium score $\geq$ median, lesions with calcium score $<$ median, and lesions without calcium. Also, we did not observe correlations between focal stent expansion versus lesion calcium score or other CTCA parameters quantitatively describing calcium deposits (Table 4). Diffuse stent expansion was similar in all 3 lesion subgroups: $92 \pm 30 \%$ vs. 85 $\pm 30 \%$ vs. $93 \pm 38 \%, p=0.7$, respectively, for lesions with calcium score $\geq$ median, lesions with calcium score $<$ median, and lesions without calcium. The lesion calcium score, total length of calcium deposits, and maximal calcium area and thickness did not correlate with diffuse stent expansion (Table 4).

Frequency of acute stent malapposition (3 (13\%) vs. $4(17 \%)$ vs. $3(23 \%), p=0.7)$, tissue prolapse (5 (21\%) vs. $2(9 \%)$ vs. $1(8 \%), p=0.2)$ and stent edge dissections (3 (13\%) vs. 1 (4\%) vs. $1(8 \%), p=0.5)$ (respectively for lesions with calcium score $\geq$ median, lesions with calcium score $<$ median, and lesions without calcium) were similar in all 3 lesion subgroups.

\section{Discussion}

The main findings of the current study were as follows. The presence and magnitude of calcium identified by CTCA were not related to the final stent expansion. Reference segments adjacent to the stent edges contained a larger plaque burden in lesions with a larger amount of calcium. Lesions with more extensive calcifications by CTCA more likely required post-dilation and higher balloon pressures before optimal angiographic results were obtained.

The CTCA is a non-invasive imaging modality that allows assessment of both the vessel lumen and coronary plaque composition and, especially, accurate quantification of calcium deposits sharing some features of IVUS [9]. Moreover, due to signal loss behind the leading edge of calcified plaque, IVUS may not evaluate the total area and thickness of calcium, which can be relatively easily quantified by CTCA. Moreover, plaque composition as 
Table 2. Comparison of IVUS findings after stent implantation in lesion with regards to the lesion calcium score

\begin{tabular}{lcccc} 
Variable & $\begin{array}{c}\text { Lesion calcium } \\
\text { score }=0(n=13)\end{array}$ & $\begin{array}{c}\text { Lesion calcium } \\
\text { score }<\text { median }(n=23)\end{array}$ & $\begin{array}{c}\text { Lesion calcium } \\
\text { score } \geq \text { median }(n=24)\end{array}$ & Value of $p$ \\
\hline Minimal stent area $\left[\mathrm{mm}^{2}\right]$ & $6.1 \pm 2.2$ & $6.4 \pm 2.4$ & $6.1 \pm 1.7$ & 0.9 \\
\hline Mean stent area $\left[\mathrm{mm}^{2}\right]$ & $7.2 \pm 2.3$ & $7.5 \pm 2.4$ & $7.3 \pm 1.7$ & 0.9 \\
\hline Proximal reference lumen area $\left[\mathrm{mm}^{2}\right]$ & $9.5 \pm 4.4$ & $11.5 \pm 3.7$ & $9.7 \pm 2.9$ & 0.18 \\
\hline Proximal reference EEM area $\left[\mathrm{mm}^{2}\right]$ & $14.4 \pm 4.3$ & $19.8 \pm 5.9$ & $18.2 \pm 4.5$ & 0.017 \\
\hline Proximal reference plaque burden [\%] & $34 \pm 18$ & $41 \pm 9$ & $47 \pm 12$ & 0.02 \\
\hline Distal reference lumen area $\left[\mathrm{mm}^{2}\right]$ & $8.2 \pm 3.4$ & $8.6 \pm 3.1$ & $7.9 \pm 2.2$ & 0.7 \\
\hline Distal reference EEM area $\left[\mathrm{mm}^{2}\right]$ & $12.7 \pm 4.1$ & $14.5 \pm 5.6$ & $3.4 \pm 4.3$ & 0.5 \\
\hline Distal reference plaque burden [\%] & $33 \pm 11$ & $38 \pm 16$ & & 0.5
\end{tabular}

EEM - external elastic membrane

assessed by CTCA may predict procedural complications such as myocardial infarction [10]. The current study examines the relation between plaque calcification by CTCA and the outcome of stent implantation by IVUS. Previous IVUS data on the possible relation between presence and extent of calcium deposits and stent expansion are conflicting. Albrecht et al. found that calcified lesions interfered with optimal stent deployment [1]. Vavuranakis et al. showed that stent expansion was inversely correlated with the extent of calcifications [2]. However, our results are in line with more recent IVUS analyses by Costa et al., who found no relation between either calcium arc or length measured by pre-intervention IVUS and stent expansion [3]. Of note, mean focal stent expansion achieved in our study was approximately $70 \%$ and approximately $25 \%$ of lesions had an MSA below the threshold of $4.5 \mathrm{~mm}^{2}$ despite optimal angiographic results. These findings are also in line with the study by Costa et al. and highlight the potential role of IVUS guidance during stent implantation even if angiographic results of the procedure look satisfactory.

We found a larger reference segment plaque burden in lesions with a higher calcium score. This is in line with reports from IVUS studies showing a positive correlation between calcium and plaque burden [11, 12]. However, as our study IVUS was performed only after stent implantation, we cannot exclude that a larger reference plaque burden was caused by larger plaque shifts after stent implantation into calcified lesions.

The results of studies by Kass et al. and LaBounty et al. and our previous reports showed that evaluation of lesion length and diameter derived from CTCA may beneficially influence stent size selection, procedure extent, and acute results as assessed by IVUS [4, 13-15]. Previous IVUS studies have also shown that a larger reference segment plaque burden was associated with increased risk of stent thrombosis [16]. Therefore, a higher lesion calcium score identified by CTCA may also influence the decision about IVUS guidance in order to ensure adequate lesion coverage. Our findings of positive correlations between maximal calcium area and maximal balloon pressure and
Table 3. Comparison of calcium deposit features according to the minimal stent area

\begin{tabular}{|c|c|c|c|}
\hline Variable & $\begin{array}{c}\text { Lesions with } \\
\text { MSA }<4.5 \mathrm{~mm}^{2} \\
(n=15)\end{array}$ & $\begin{array}{c}\text { Lesions with } \\
\text { MSA } \geq 4.5 \mathrm{~mm}^{2} \\
(n=45)\end{array}$ & Value of $p$ \\
\hline $\begin{array}{l}\text { Lesion calcium } \\
\text { score }\end{array}$ & $45.2(3.3-165.0)$ & $27.6(3.7-78.3)$ & 0.6 \\
\hline $\begin{array}{l}\text { Total length of all } \\
\text { calcium deposits [mm }\end{array}$ & $\begin{array}{l}6.1(2.0-10.0) \\
n]\end{array}$ & $5.5(2.0-11.0)$ & 0.9 \\
\hline $\begin{array}{l}\text { Maximal calcium } \\
\text { area }\left[\mathrm{mm}^{2}\right]\end{array}$ & $2.2(1.1-2.9)$ & $2.8(1.0-5.1)$ & 0.3 \\
\hline $\begin{array}{l}\text { Maximal calcium } \\
\text { thickness [mm] }\end{array}$ & $1.5(1.0-2.0)$ & $1.4(1.0-2.0)$ & 0.5 \\
\hline
\end{tabular}

Table 4. Correlation between quantitative CTCA variables describing calcium deposits and measures of stent expansion by IVUS

\begin{tabular}{lcc} 
Variable & $\begin{array}{c}\text { Focal stent } \\
\text { expansion [\%] }\end{array}$ & $\begin{array}{c}\text { Diffuse stent } \\
\text { expansion [\%] }\end{array}$ \\
\hline Lesion calcium score & $R=-0.13, p=0.3$ & $R=-0.05, p=1.0$ \\
\hline $\begin{array}{l}\text { Total length of all } \\
\text { calcium deposits [mm] }\end{array}$ & $R=-0.01, p=0.9$ & $R=0.02, p=0.9$ \\
\hline $\begin{array}{l}\text { Maximal calcium } \\
\text { area [mm²] }\end{array}$ & $R=0.06, p=0.6$ & $R=0.04, p=0.7$ \\
\hline $\begin{array}{l}\text { Maximal calcium } \\
\text { thickness [mm] }\end{array}$ & $R=-0.07, p=0.6$ & $R=-0.3, p=0.8$ \\
\hline
\end{tabular}

between lesion calcium score and frequency of post-dilation were also in line with the results of Vavuranakis et al. and further support the concept that CTCA data should be used, if available, for preliminary planning of the stent implantation procedure $[2,4]$.

The major limitation of our study was the relatively small sample size and the fact that the results may not apply to heavily calcified lesions.

\section{Conclusions}

Lesions with a higher calcium score had a larger reference plaque burden after stent implantation and were more likely to require post-dilation with higher balloon 
pressure, but final stent expansion as assessed by IVUS was not affected provided an angiographically optimal result was achieved.

\section{References}

1. Albrecht D, Kaspers S, FüssI R, et al. Coronary plaque morphology affects stent deployment: assessment by intracoronary ultrasound. Cathet Cardiovasc Diagn 1996; 38: 229-235.

2. Vavuranakis M, Toutouzas K, Stefanadis C, et al. Stent deployment in calcified lesions: can we overcome calcific restraint with highpressure balloon inflations? Catheter Cardiovasc Interv 2001; 52: 164-172.

3. Costa J, Mintz G, Carlier S, et al. Intravascular ultrasound assessment of drug-eluting stent expansion. Am Heart J 2007; 153: 297-303.

4. Pregowski J, Kepka C, Kruk M, et al. Comparison of usefulness of percutaneous coronary intervention guided by angiography plus computed tomography versus angiography alone using intravascular ultrasound end points. Am J Cardiol 2011; 108: 1728-1734.

5. Mintz GS, Nissen SE, Anderson WD, et al. ACC clinical expert consensus document on standards for the acquisition, measurement and reporting of intravascular ultra- sound studies: a report of the American College of Cardiology Task Force on Clinical Expert Consensus Documents (Committee to Develop a Clinical Expert Consensus Document on Standards for Acquisition, Measurement and Reporting of Intravascular Ultrasound Studies [IVUS]). J Am Coll Cardiol 2001; 37: 1478-1492.

6. Kim SW, Mintz GS, Ohlmann P, et al. Frequency and severity of plaque prolapse within Cypher and Taxus stents as determined by sequential intravascular ultrasound analysis. Am J Cardiol 2006; 98: 1206-1211.

7. Hong MK, Mintz GS, Lee CW, et al. Incidence, mechanism, predictors, and long-term prognosis of late stent malapposition after bare-metal stent implantation. Circulation 2004; 109: 881-886.

8. Sheris SJ, Canos MR, Weissman NJ. Natural history of intravascular ultrasound-detected edge dissections from coronary stent deployment. Am Heart J 2000; 139: 59-63.

9. Okabe T, Mintz GS, Weigold WG, et al. The predictive value of computed tomography calcium scores: a comparison with quantitative volumetric intravascular ultrasound. Cardiovasc Revasc Med 2009; 10: 30-35.

10. Uetani T, Amano T, Kunimura A, et al. The association between plaque characterization by $\mathrm{CT}$ angiography and post-procedural myocardial infarction in patients with elective stent implantation. JACC Cardiovasc Imaging 2010; 3: 19-28.

11. Tinana A, Mintz GS, Weissman NJ. Volumetric intravascular ultrasound quantification of the amount of atherosclerosis and calcium in nonstenotic arterial segments. Am J Cardiol 2002; 89: 757-760.

12. Mintz GS, Pichard AD, Popma JJ, et al. Determinants and correlates of target lesion calcium in coronary artery disease: a clinical, angiographic and intravascular ultrasound study. J Am Coll Cardiol 1997; 29: 268-274.

13. Kass M, Glover CA, Labinaz M, et al. Lesion characteristics and coronary stent selection with computed tomographic coronary angiography: a pilot investigation comparing CTA, QCA and IVUS. J Invasive Cardiol 2010; 22: 328-334.

14. LaBounty T, Sundaram B, Chetcuti S, et al. Stent size selection using 64-detector coronary computed tomography angiography: a comparison to invasive coronary angiography. Acad Radiol 2008; 15: 820-826.

15. Pregowski J, Kepka C, Kalinczuk L, et al. Comparison of intravascular ultrasound, quantitative coronary angiography, and dualsource 64-slice computed tomography in the preprocedural assessment of significant saphenous vein graft lesions. Am J Cardiol 2011; 107: 1453-1459.

16. Fujii K, Carlier SG, Mintz GS, et al. Stent underexpansion and residual reference segment stenosis are related to stent thrombosis after sirolimus-eluting stent implantation: an intravascular ultrasound study. J Am Coll Cardiol 2005; 45: 995-998. 